\title{
Bioinspired Mitigation Scheme for Cascading Failures in Farmland Wireless Sensor Networks
}

\author{
Jun Wang $\left(\mathbb{D},{ }^{1}\right.$ Zhuangzhuang Du $\mathbb{D},^{2}$ Xunyang Wang $\left(\mathbb{D},{ }^{3,4}\right.$ and Zhengkun He $\mathbb{D}^{5}$ \\ ${ }^{1}$ School of Electrical Engineering, Henan University of Science and Technology, Luoyang, Henan 471000, China \\ ${ }^{2}$ School of Agricultural Equipment Engineering, Henan University of Science and Technology, Luoyang, Henan 471003, China \\ ${ }^{3}$ Department of Applied Mathematics, Lanzhou University of Technology, Lanzhou, Gansu 730050, China \\ ${ }^{4}$ Postdoctoral Research Station in Gansu Electric Power Research Institute, Wanxin South Road, Anning District, Lanzhou, \\ Gansu 730000, China \\ ${ }^{5}$ School of Computer Science and Engineering, Central South University, Changsha, Hunan 410000, China
}

Correspondence should be addressed to Xunyang Wang; 12198114@163.com

Received 12 June 2020; Accepted 16 October 2020; Published 5 November 2020

Academic Editor: Fran ois P r s

Copyright (c) 2020 Jun Wang et al. This is an open access article distributed under the Creative Commons Attribution License, which permits unrestricted use, distribution, and reproduction in any medium, provided the original work is properly cited.

Existing mitigation strategies on wireless sensor networks (WSNs) against cascading failures cannot appropriately adapt the particular characteristics of farmland WSNs. Spider web provides a new reference for improving network invulnerability. In this study, a bionic network scheme is built based on symmetric analysis of a series of spider-web vibration transmission trials, which include networking methodology, communication rules, and load capacity model. The basic idea of this scheme is to apply the cascading-failure coping mechanism inspired by spider web into the construction and operation of farmland WSNs. We found that the link backup contributed by a topological structure and communication rules had positive effects on suppressing the spread of cascading failures. The study showed that the damages of cascading failures can be efficiently lowered by regulating the adjustment coefficient of the load capacity model. The difference between the inner-layer node failures and outer-layer node failures for network invulnerability was verified under deliberate attack circumstances. Based on these results, the proposed network scheme can be utilized to enhance the invulnerability performance of farmland WSNs.

\section{Introduction}

Wireless sensor networks (WSNs) are a distributed network system consisting of a large number of sensor nodes to wirelessly cooperate in perceiving and processing various information $[1,2]$. Due to the coupling relationship between node traffics, failure nodes will lead to malfunctions of neighboring nodes resulting in a cascading effect [3]. Cascading faults inevitably lead to topological segmentation, reduced communication connectivity, limited network coverage, and increased likelihood of network paralysis [4]. Therefore, cascading failures are an essential factor affecting network invulnerability and should be widely investigated both by theoretical analyses and experimental characterizations.

Some aspects of cascading failures in WSNs have been discussed in recent works, such as the cascade control, defense strategy, and analytical calculation of load distribution $[5,6]$. Identification and reinforcement of the critical nodes that guarantee the network functionality have been proved to have an inhibiting effect on the network disruptions produced by cascading failures $[7,8]$. However, it is still challenging to assure the accuracy of quantifying the node importance and the stability of the network topology after adding redundant nodes [9]. Predetected attack mode or dynamic topology repair has been discovered to improve the response efficiency for cascading failures $[10,11]$. Nevertheless, the targeted repairs based on the real-time detection of attacks are more feasible [12]. Furthermore, it is revealed that the initial load assignment of nodes and the load distribution strategy of fault nodes have a positive impact on avoiding the spread of cascading failures [13]. But for different application scenarios, these established load 
capacity models demonstrate notable performance differences [14].

As an innovative data acquisition method in precision agriculture, farmland WSNs can continuously monitor indispensable environmental factors for crop growth and generate remarkable labor-saving benefits to farmers [15]. Compared with other WSN applications, influenced by the agriculture production mode, farmland WSNs present unique characteristics in terms of network topology, information transmission, and load capacity [16]. First, due to the largescale monitoring area, multiple transmission constraints, and limited cost budget, it is essential to introduce multilevel relay nodes to achieve reliable data transfer with low-density deployment, and the network topology displays the feature of hierarchical clustering $[17,18]$. The combined factors of node mobility, network heterogeneity, and chain-directed transmission dramatically boost the dynamic uncertainty of network behavior, which make farmland WSNs exhibit substantial topological variability [19]. Besides, the pattern of multinode collaborative monitoring causes overlap and interaction between interlayer nodes, intercluster nodes, and communication links, resulting in network transmission with both interlayer coupling association and intersecting coupling association [20]. Lastly, the entire network shows multilevel differences in load capacity, as a consequence of differences in detecting tasks and monitoring frequencies for nodes [21]. In summary, farmland WSNs are a complex network system with the characteristics of coupling and vulnerability, and a few node or link faults are prone to provoke cascading failure disasters. The current mitigation techniques of cascading failures have the shortcoming of overidealized assumptions and sole coping means [22]. It is an urgent requirement to develop a specialized countermeasure scheme for farmland WSNs by systematic consideration of cascading failure mechanism and mitigation measures.

Over hundreds of millions of years, the spider web has evolved an elegant, ultralight, and destruction-resistant structure [23]. The previous studies tested the hypothesis that the orb spider web has a stunning similarity in topological structures and component functions with farmland WSNs and can be used to promote the network capability against cascading failures [24]. The existing research focuses on the invulnerability performance of artificial spider-web topology, but the enlightenment of vibration transmission characteristics of destructed spider web on the alleviation of cascading failures has not been discussed [25]. By investigating the transmission mechanism of spider-web vibration, we can develop a bioinspired network scheme for improving the invulnerability of farmland WSNs.

The objective of this study is to build a bionic network scheme of cascading-failure mitigation for farmland WSNs inspired by the vibration transmission reactions of artificial spider web under various destruction cases. The major contributions of this study are summarized as follows:

(1) To properly characterize the response process of spider web to cascading damages, an experimental method based on artificial spider-web vibration testbed is proposed.
(2) A network scheme comprised of networking methodology, communication rules, and load capacity model is presented in the face of random and deliberate attacks on nodes.

(3) Through extensive trials, the soundness and effectiveness of the network scheme against cascading failures are verified. The impact of scheme parameters on invulnerability is also been explored.

The remainder of this paper is arranged as follows: the damage experiments of artificial spider web and the suppression mechanism of cascading propagation are given in Section 2; the details of the proposed network scheme for farmland WSNs are displayed in Section 3; the evaluations on the performances of network scheme and the impacts of key parameters are exhibited in Section 4; finally, conclusions are shown in Section 5.

\section{Artificial Spider-Web Experiments and Mechanism Analysis}

We use the developed artificial spider-web vibration testbed (Figure 1) to clarify the cascading spread characteristics of spider web after damage to partial components [26]. In the experiments, a rubber ball with a mass of $20 \mathrm{~g}$ and a diameter of $50 \mathrm{~mm}$ is utilized as the excitation source. Each radius line from the central node and the horizontal connection line of each layer that encircled the central node are defined as a radial line and a spiral line, respectively. The intersection of a radial line and a spiral line is designated as a node. Moreover, the radial line between adjacent spiral lines is described as a radial path, and the spiral line between adjacent radial lines is identified as a spiral path. Moreover, radial paths, spiral paths, and nodes constitute the artificial spider-web topology.

2.1. Node Damage. Figure 2 indicates the variations in peakto-peak values of longitudinal vibrations of 51 nodes on radial lines $6-10$ before and after cutting off four nodes at intersections of radial lines 6-7 and spiral lines $1-2$. It can be noticed that the vibrations at these nodes decrease from the inner layers to the outer layers without changing the attenuation characteristics of nodes on a radial line, except the vibrations of each node increase insignificantly. The peak-topeak values of node vibrations in areas A1 and B1 are 1.20 and 1.10 times of that in the complete web, and the vibrations of nodes in areas $C 1, D 1$, and $D 2$ are consistent with that of the complete web. These results show that the node failures have a slight effect on the vibration transmission of spider web, and the affected area is within the adjacent layers, manifesting that the cascading propagation range is limited. Furthermore, in trapezoid area A1 and triangle area B1, the amplitude increments of vibrations for six nodes are the same, showing that the triangle and trapezoid structures can play the role of dissipation and inhibition of cascading spread.

2.2. Radial Line Damage. Figure 3 displays the variations in peak-to-peak values of longitudinal vibrations of nodes on radial lines 6-10, 7-11, and 8-12 as cutting out radial line 5, 




(a)



(b)

Figure 1: Artificial spider-web vibration testbed.

radial lines 5-6, and radial lines 5-7, respectively. As can be seen from Figures 3(a) and 3(b), with the elimination of radial line 5 , the node vibrations on radial lines 6-10 dramatically enhance. In areas $A 1, B 1, C 1, \mathrm{D} 1$, and $E 1$, the peakto-peak values rise to $2.0,1.9,1.8,1.6$, and 1.4 times of that in the complete web on average, respectively. For areas $F 1$ and $G 1$, the values grow to 1.3 and 1.2 times of that in the complete web on average, respectively. It can be concluded that the closer to the failure positions, the more intense the vibrations of nodes on the radial lines, and vice versa. In Figures 3(c) and 3(d), with radial lines 5-6 being destroyed, the increments of peak-to-peak values of nodes on radial lines 7-11 are observable. Compared with the corresponding positions of the complete web, the average peak-to-peak values increase by $1.2,1.0,0.8,0.6$, and 0.6 times in areas $A 1$, $B 1, C 1, D 1$, and $E 1$, raise by $1.4,1.2,0.4$, and 0.3 times in areas $B 2, C 2, D 2$, and $E 2$, and boost by 1.1 and 0.8 times in areas D3 and E3. It can be found that, with the increase in damaged radial lines, the coupling relationship between layers and adjacent radial lines causes the cascading spread of the radial-line failure effect. As shown in Figures 3(e) and $3(\mathrm{f})$, when the number of damaged radial lines jumps to 3 , the average peak-to-peak values of nodes in areas $A 1, B 1, C 1$, $D 1$, and $E 1$ enhance by $1.31,1.07,0.80,0.61$, and 0.57 times and that in areas $A 2, B 2, C 2, D 2$, and $E 2$ grow by 2.30, 1.83, $0.60,0.33$, and 0.31 times, respectively. It can be recognized that the nodes of layers $1-5$ with dense deployment mutually undertake the cascading propagation generated by radial line damages, which results in the vibrations lower than that of nodes at layers 6-11 with sparse deployment.

The results show that, with the growth of the number of damaged radial lines, the node vibrations on the remaining radial lines raise and obey the principle of nearby sharing, which is identical to the phenomenon of cascading-failure spread in WSNs. Besides, the difference between the vibration tracking areas increase with the increment of the number of failed radial lines. Furthermore, the increasing deviations of node vibrations in these triangle and trapezoid 



(a)

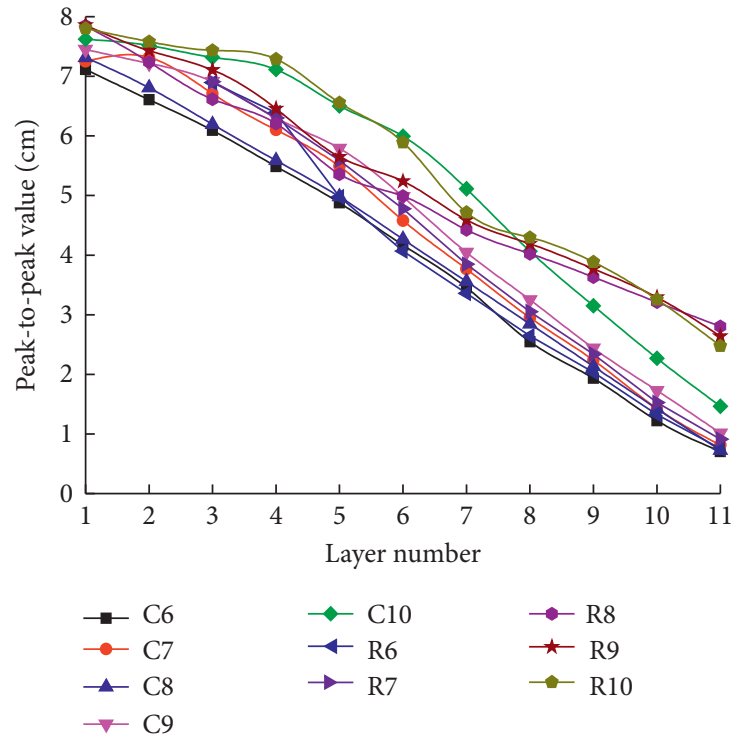

(b)

Figure 2: Differences in peak-to-peak values of longitudinal vibrations for nodes on radial lines 6-10 before and after node damages. (a) Analysis area division. $Z 1$ denoted the node failure area, and $A 1, B 1, C 1, D 1$, and $D 2$ expressed a vibration tracking area, respectively. (b) Peak-to-peak values of longitudinal vibrations of the tracked nodes. C6-10 depicted the vibrations of 55 nodes on radial lines 6-10 during the complete web, and R6-10 represented the vibrations of 51 nodes on radial lines 6-10 after the node failures, respectively.



(a)

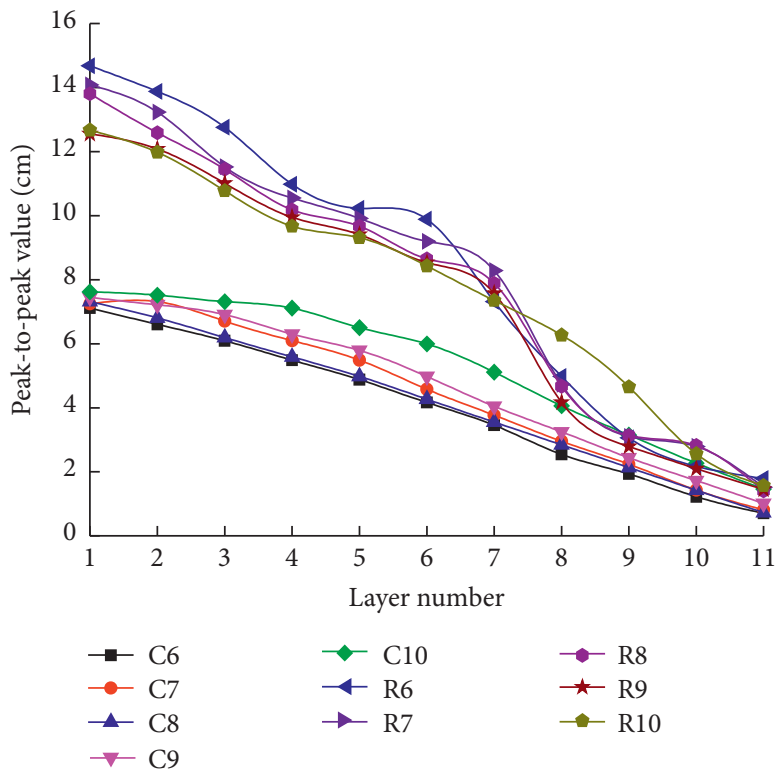

(b)

Figure 3: Continued. 


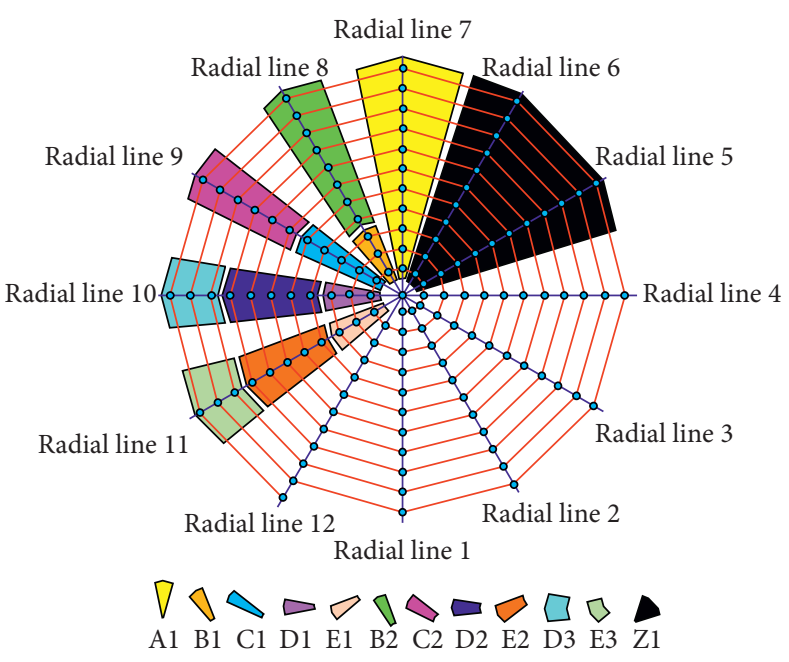

(c)

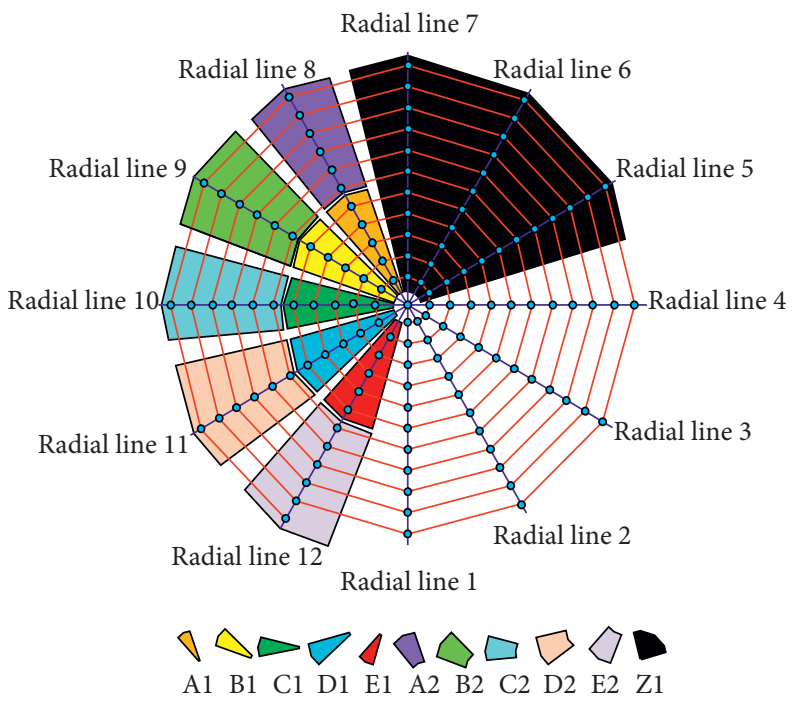

(e)

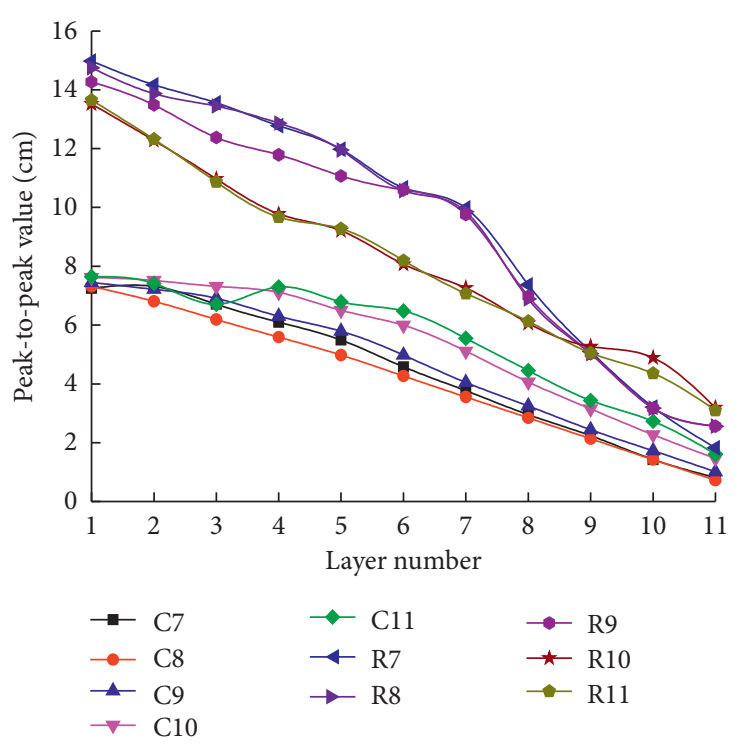

(d)

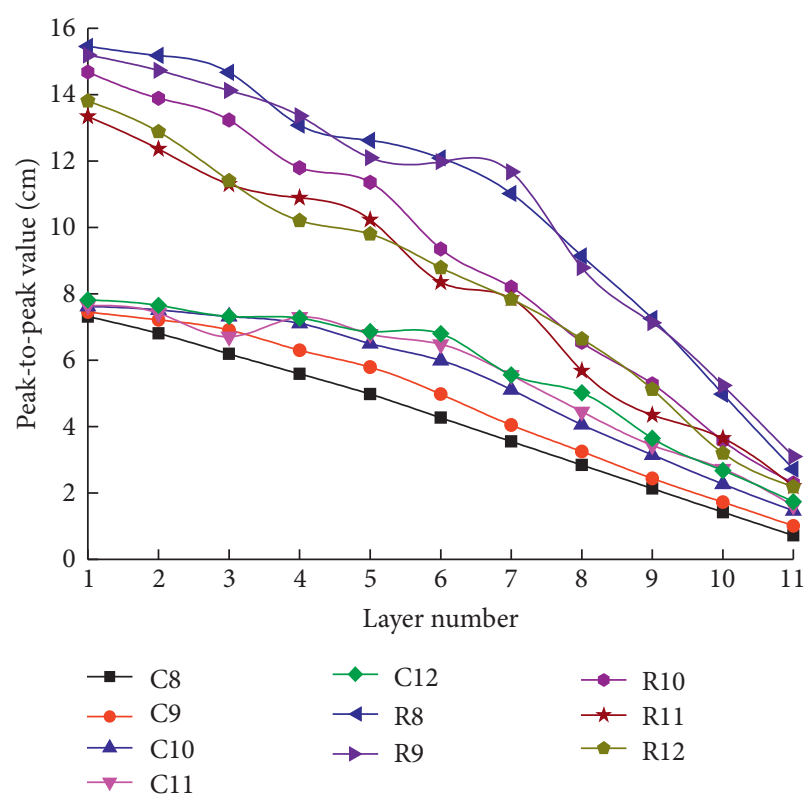

(f)

Figure 3: Variations in peak-to-peak values of longitudinal vibrations for nodes on radial lines 6-10, radial lines 7-11, and radial lines 8-12 before and after radial line damages: (a) division of tracking areas at the failure of radial line 5; (b) peak-to-peak values of longitudinal vibrations of the tracked nodes on radial lines 6-10; (c) distribution of tracking areas at the failure of radial lines 5-6; (d) peak-to-peak values of longitudinal vibrations of the tracked nodes on radial lines 7-11; (e) allocation of tracking areas under the damage condition of radial lines $5-7$; (f) peak-to-peak values of longitudinal vibrations of the tracked nodes on radial lines 8-12.

areas are comparatively small, which illustrates that the triangle and trapezoid structures can accomplish the hierarchical reduction of vibrations in the inner layers.

2.3. Spiral Line Damage. Figure 4 manifests the differences in peak-to-peak values of longitudinal vibrations for the nodes on radial lines 8-9 under the condition that the spiral lines 1-5 are removed layer by layer. It can be observed that as the spiral lines $1-5$ are destroyed one by one, the node vibrations of radial lines $8-9$ strengthen gradually. The average peak-to-peak values of nodes on radial line 8 reach $1.10,1.30,1.46,1.56$, and 1.70 times of the complete web, respectively. The average peak-to-peak values of nodes on radial line 9 become 1.16, 1.30,1.39, 1.51, and 1.62 times of the complete web, respectively. It is obvious that the number of broken spiral lines is positively correlated with the vibration intensity of the tracked nodes. Meanwhile, the influence of the damaged spiral lines on the cascading propagation lies between node failures and radial-line 


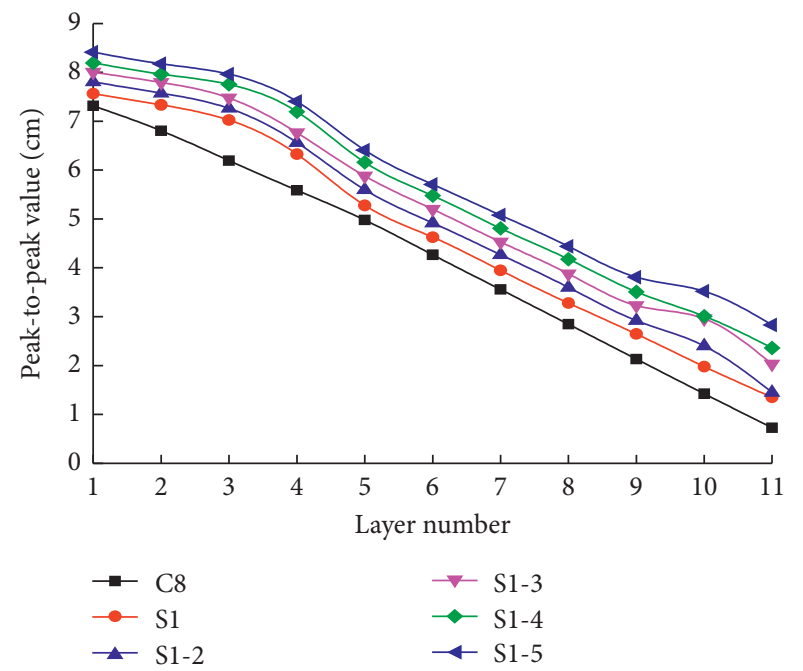

(a)

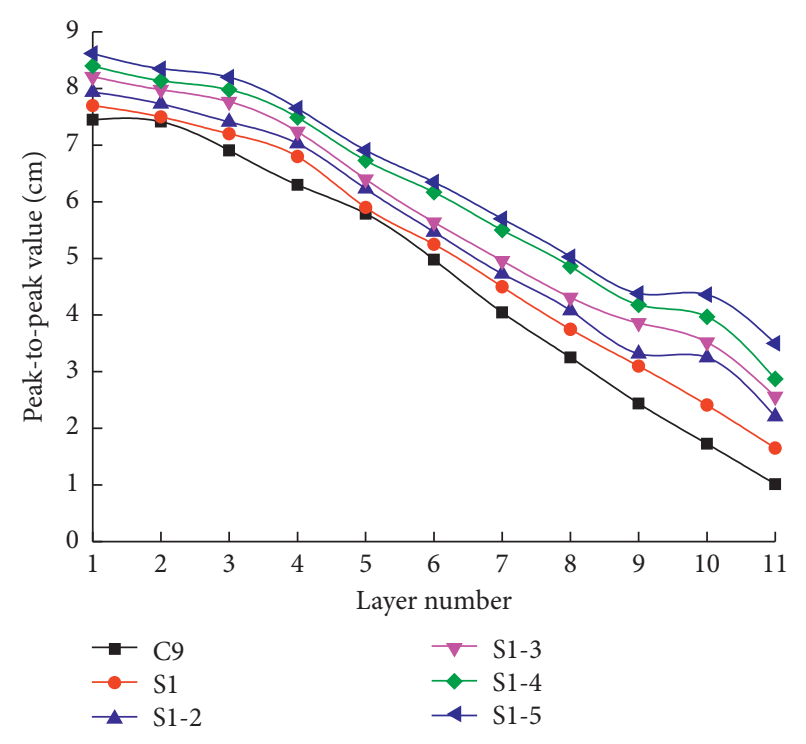

(b)

FIGURE 4: Changes in peak-to-peak values of longitudinal vibrations for nodes on radial lines 8-9 under situation of successive failures of spiral lines 1-5: (a) peak-to-peak values of longitudinal vibrations of nodes on radial line 8; (b) peak-to-peak values of longitudinal vibrations of nodes on radial line 9.

failures, indicating that the spiral lines only serve as an aid to the vibration transmission.

By analyzing the influences of different component failures on the vibration distributions, we can summarize the mitigation mechanism of cascade failures in the artificial spider web as follows:

(1) The hierarchical redundancy arrangement of nodes and the internal-external differentiation of node densities can share the burden of inner-layer nodes to cope with the cascading spread.

(2) In the case of node and path damages, the peripheral nodes and paths will carry the vast majority of vibration variations, and the topological structures of triangles and trapezoids can reduce the cascading diffusion effect by using the hierarchical weakening process.

(3) Except for the central node and the outermost nodes, all the other nodes simultaneously connect with the four ambient nodes through radial paths and spiral paths, which has distinct feature of link backup. In addition, radial paths shoulder more transmission responsibilities than spiral paths.

Therefore, on the basis of the inspirations of artificial spider-web topology against cascading failures (topological structures, node deployment mode, and link backup mechanism), combining with the characteristics of farmland WSNs, we should be able to develop a bionic network scheme to alleviate the cascading effect for potential network attacks. Furthermore, the artificial spider-web topology has been proven to be more invulnerable than other traditional topologies in harsh network environments, with the possibility for improving the capability of farmland WSNs against cascading failures [27].

\section{Network Scheme for Mitigating the Cascading Failures}

In this section, through characterizing the mitigation process of cascading spread in artificial spider web, we describe how to establish a comprehensive network scheme for cascade control guidance of farmland WSNs by assembling networking methodology, communication rules, and load capacity model.

3.1. Networking Methodology. To discuss the network formation procedure, the assumptions are presented as follows:

(1) Each node in the network has a unique physical coordinate, the physical coordinates of the sink node are set to $(0,0)$, and the physical coordinates of other nodes are determined by the communication with the sink node.

(2) The sink node has the capacity to communicate throughout the network, and other nodes barely interact with nodes in the adjacent areas.

(3) The nodes are in gradient distribution according to the distance from the sink node, and the deployment density gradually increases from the inside out to ensure network coverage. In addition, no isolated nodes exist in the network, and every communication link is symmetrical.

(4) The network adopts carrier monitoring multiple access with collision avoidance (CSMA/CA) protocol to prevent channel conflicts during data transmission [28].

In this premise, we propose the networking methodology of farmland WSNs to perform the hierarchical clustering 
routing protocol and topological frame structures composed of triangle and trapezoid units, which are motivated by spider web. The detailed networking steps are listed as follows:

(1) The layer number of the sink node is initialed as 0 . The sink node broadcasts the networking packets to the whole network for discovering child nodes. All nodes that have received packets are regarded as child nodes of the sink node and automatically estimate individual physical coordinates by measuring the distances and orientations from the sink node [29]. Afterwards, the physical coordinates of each child node are added into the corresponding routing table (Table 1).

(2) Each child node acquires its layer number by Euclidean distance from the sink node and stores the layer number in the routing table. The layer number coding of child node positively correlates with the distance from the sink node, and the interlayer spacing division can be regulated to balance the number of nodes in each layer. Followed by the assignments of layer numbers, the sink node is indicated as $N_{0}$. Besides, the nodes of the $n$th layer uniformly are coded in the clockwise direction according to orientations and expressed as $N_{n-1}, N_{n-2}, N_{n-3}$, etc.

(3) Once the layer numbers and physical coordinates of the whole-network nodes are fixed, the networking frames of sink node are sent out layer by layer starting from the child nodes in the first layer. Apparently, the nodes not only transmit the networking frames to the neighboring child nodes in different layers but also receive the frames from other child nodes in the same layer. By comparing its layer number with the layer number of source nodes, each child node receiving the network frames can be classified into three categories as follows:

(a) The two-layer numbers were equal, it means that the sender and the receiver are in the same layer, and the distance between the two child nodes is recorded. If the networking process of the first layer is accomplished, the two senders closest to each receiver will be registered as nodes of transverse links in the routing table of receiver.

(b) The layer number of the receiver is less than that of the sender; it indicates that the receiver belongs to the upper layer, and then. the distance between the two nodes is noted. At the end of the networking process of the first layer, the node with the smallest distance from the sender among all the receiving nodes is designated in the routing table as the radial-link node of the sender.

(c) The number of layers of the receivers is higher than that of the senders, and no operation will be done.

(4) Through iterating step (3), all the layers can finish the networking processes in turn, and a topological
TABLE 1: Routing table for child node.

\begin{tabular}{lcc}
\hline Index labels & Categories & Lengths \\
\hline 1 & Layer number & 8 \\
2 & Physical coordinates & 32 \\
3 & Node of radial link & 8 \\
4 & Node 1 of transverse link & 8 \\
5 & Node 2 of transverse link & 8 \\
\hline
\end{tabular}

framework modeled from spider web is developed (Figure 5).

As shown in Figure 5, the farmland WSNs topology is formed of numerous topological units of triangles and trapezoids. The basic triangle structure is constituted of the child node in the first layer and its transverse-link node, the sink node, and the corresponding links. The fundamental trapezoid structure is included of any two adjacent-layer nodes with a radial link, their transverse-link nodes, and related links. It can be perceived that the triangle and trapezoid structures in the same area have interlayer coupling and intersecting coupling relations, and the radial-link coupling relationship exists between adjacent triangle or trapezoid structures.

3.2. Communication Rules. The link backup and differential distribution of load are the core methods of artificial spiderweb topology to overcome cascading failures. Thus, the communication rules of the farmland WSNs based on the invulnerability characteristics of spider web are defined as follows:

(1) In the network, a nonsink node connects with three neighboring nodes by transmission logic, including a radial-link node in the upper layer and two transverse-link nodes in the same layer. The transfer probability of radial link and transverse links is defined as $\alpha$ and $\beta(1 \geq \alpha>\beta>0$ and $\alpha+2 \beta=1)$, respectively, and that of a failure link is set as 0 .

(2) If the node traffic exceeds its load capacity, the node is assessed to be a failure node, and the associated outer node transmits data to the sink node through its transverse-link nodes.

(3) In the occurrence of a node failure, all the links connect with it failed, and data receiving and delivering cannot be carried out. The function of the failed node is assumed by its transverse-link nodes. Assuming that the communication reliability between a certain node and the three linked nodes is $\lambda$ $(0 \leq \lambda \leq 1)$, the transfer probabilities and transmission success rates of the node under different circumstances are assigned in Table 2.

(4) At the time that the ratio of the failure nodes to the whole-network nodes surpasses the upper limit of node failure rate $\gamma$, the sink node will rebroadcast the networking request. 


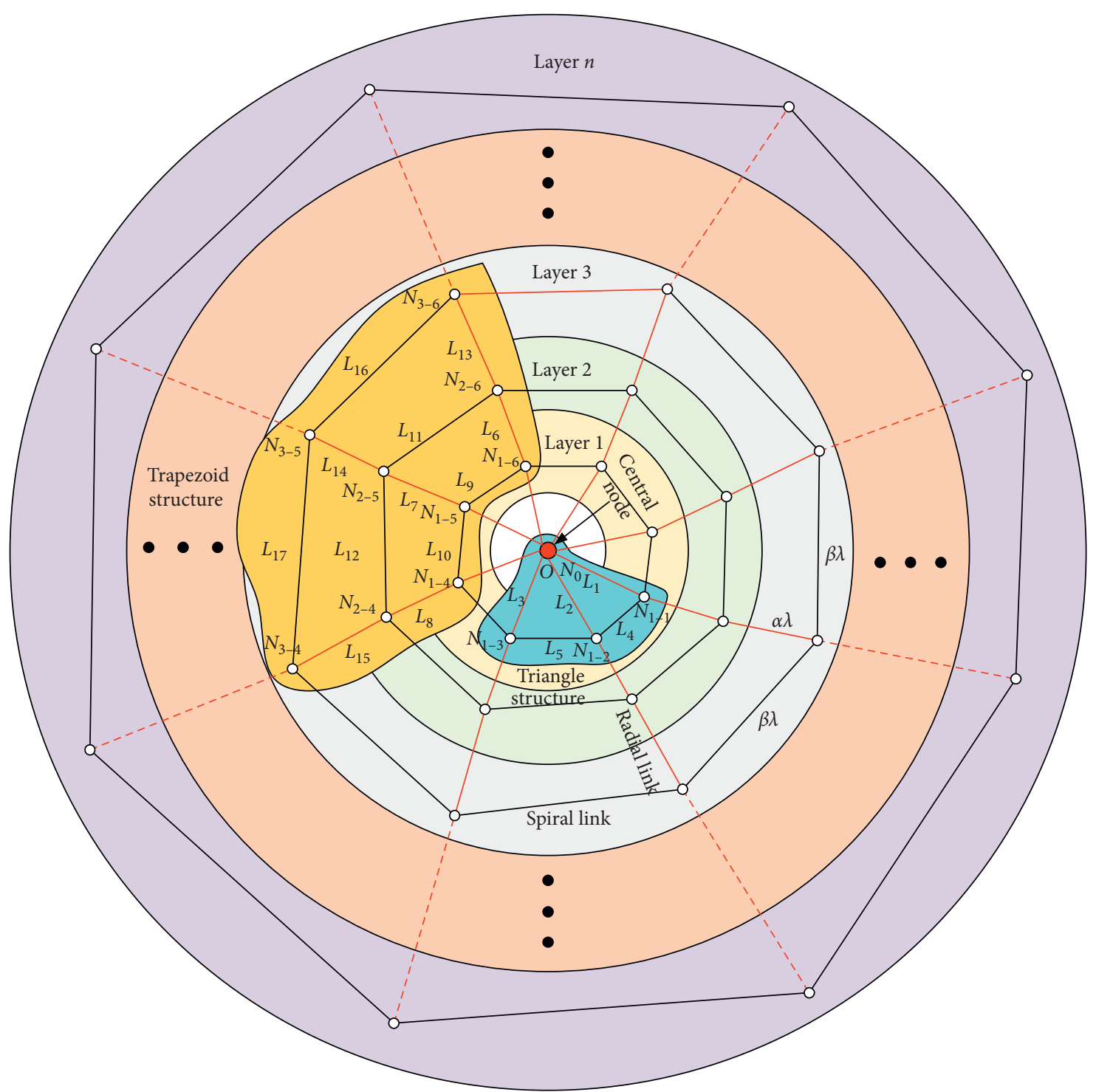

FIGURE 5: Bionic networking for farmland WSNs based on artificial spider-web topology.

TABLE 2: Node communication settings in various conditions.

\begin{tabular}{lccc}
\hline Categories & Descriptions & Transfer probability & Transmission success rate \\
\hline Normal condition & Trouble-free operation of nodes and links & $\alpha+2 \beta=1$ & $\alpha \lambda+2 \beta \lambda$ \\
& Failure of radial-link node & $2 \beta=1$ & $2 \beta \lambda$ \\
Node failures & Failures of both transverse-link nodes & $\alpha=1$ & $\alpha \lambda$ \\
& Failures of radial-link node and a transverse-link node & $\beta=1$ & 0 \\
& Failures of radial-link node and transverse-link nodes & 0 & $2 \beta \lambda$ \\
Link failures & Failure of radial link & $\alpha=1$ & $\alpha \lambda$ \\
& Failures of both transverse links & $\beta=1$ & $\beta \lambda$ \\
\hline
\end{tabular}

Furthermore, we select a triangle structure and a trapezoid structure to analyze the cascading-failure mitigation means of the particular topological structures stimulated by artificial spider-web topology.

Figure 6 shows the triangle structure comprised of nodes $N_{0}, N_{1-1}-N_{1-3}$, radial links $L_{1}-L_{3}$, and transverse links $L_{4}-L_{5}$.
In traditional point-to-point mode, the data transmission between nodes is entirely dependent on the link between them. In contrast, the radial and transverse links of nodes in the triangle structure can both participate in data transfer, and the link backup improves the invulnerability of the network. For instance, the transmission success rate between 


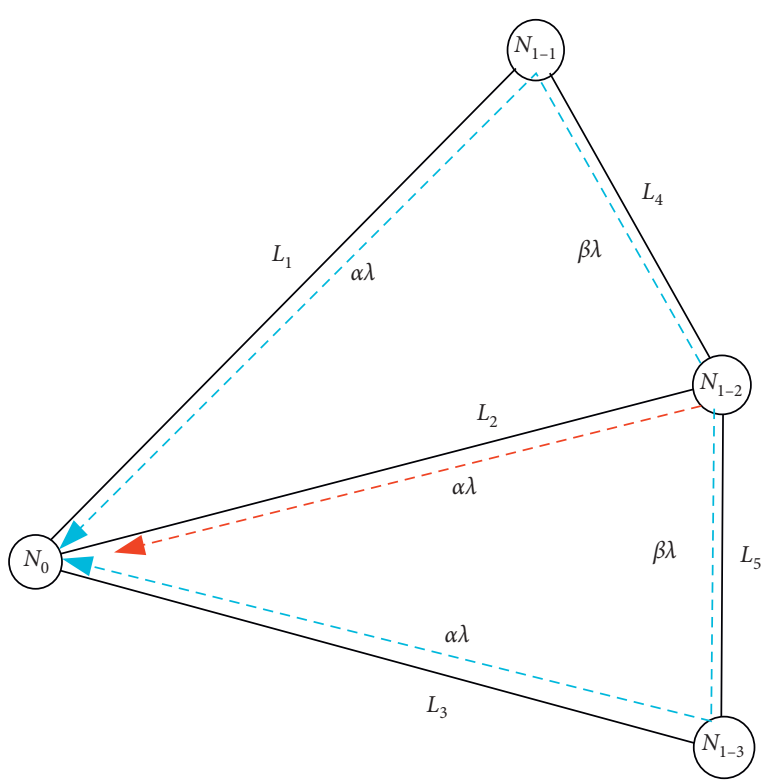

Figure 6: Triangle structure.

$N_{1-2}$ and $N_{0}$ is $\alpha \lambda+2 \beta \alpha \lambda$ under normal case, while in the situation of radial link $L_{2}$ damaged, $N_{1-2}$ can transfer data to $N_{0}$ through the relay nodes $N_{1-1}$ and $N_{1-3}$, and the transmission success rate becomes $2 \beta \alpha \lambda$.

Figure 7 displays the trapezoid structure composed of nodes $N_{1-4}-N_{1-6}, N_{2-4}-N_{2-6}$, and $N_{3-4}-N_{3-6}$ and links $L_{6}-L_{17}$. The transmission success rates of $N_{3-5} \longrightarrow N_{2-5}$, $N_{2-5} \longrightarrow N_{1-5}$, and $N_{3-5} \longrightarrow N_{1-5}$ are computed in state of the normal condition, $L_{14}$ failed, $N_{2-5}$ damaged, $L_{7}$ disabled, and $N_{1-5}$ corrupted, respectively.

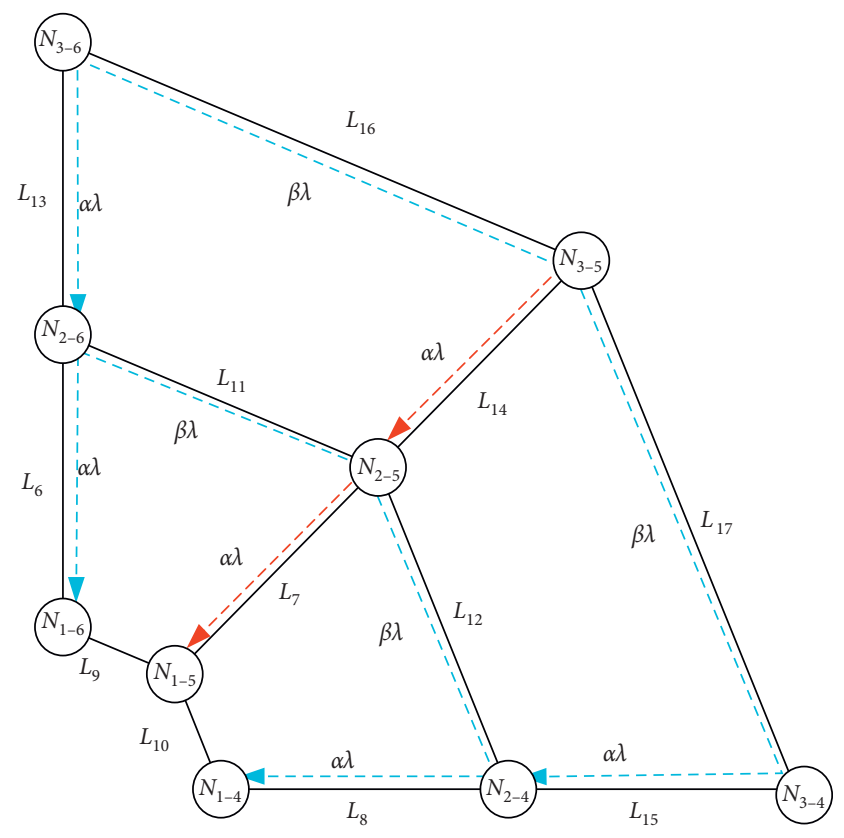

FIgURE 7: Trapezoid structure.

It can be seen that the failure of nodes or links at different positions on the radial link will not cause the communication interruption depending on the trapezoidal structure. The analysis indicates that the trapezoid structure can lessen the cascading-failure effect of failed nodes or links to guarantee data transmission by utilizing link backup.

Normal condition:
$L_{14}$ failed:

$$
\left\{\begin{array}{l}
P\left(N_{3-5} \longrightarrow N_{2-5}\right)=2 \beta \cdot \alpha \cdot \lambda^{2}, \\
P\left(N_{2-5} \longrightarrow N_{1-5}\right)=2 \alpha \cdot \lambda+2 \beta \cdot \alpha \cdot \lambda^{2}, \\
P\left(N_{3-5} \longrightarrow N_{1-5}\right)=4 \beta \cdot \alpha \cdot \lambda^{2} \cdot\left(\alpha \cdot \lambda+\alpha \cdot \beta \cdot \lambda^{2}\right) .
\end{array}\right.
$$

$N_{2-5}$ damaged:

$$
\left\{\begin{array}{l}
P\left(N_{3-5} \longrightarrow N_{2-4} \text { and } N_{2-6}\right)=2 \alpha \cdot \beta \cdot \lambda^{2}, \\
P\left(N_{2-4} \text { and } N_{2-6} \longrightarrow N_{1-5}\right)=2 \alpha \cdot \lambda, \\
P\left(N_{3-5} \longrightarrow N_{1-5}\right)=2 \beta \cdot \alpha^{2} \cdot \lambda^{3} .
\end{array}\right.
$$

$L_{7}$ disabled:

$$
\left\{\begin{array}{l}
P\left(N_{3-5} \longrightarrow N_{2-5}\right)=\alpha \cdot \lambda+2 \beta \cdot \alpha \cdot \lambda^{2}, \\
P\left(N_{2-5} \longrightarrow N_{1-5}\right)=\alpha \cdot \lambda+2 \beta \cdot \alpha \cdot \lambda^{2}, \\
P\left(N_{3-5} \longrightarrow N_{1-5}\right)=\alpha^{2} \cdot \lambda^{2}+\alpha \cdot \lambda \cdot\left(\alpha \cdot \lambda+2 \beta \cdot \alpha \cdot \lambda^{2}\right)+2 \alpha \cdot \beta \cdot \lambda^{2}\left(\alpha \cdot \lambda+\beta \cdot \alpha \cdot \lambda^{2}\right) .
\end{array}\right.
$$

$$
\left\{\begin{array}{l}
P\left(N_{3-5} \longrightarrow N_{2-5}\right)=\alpha \cdot \lambda+2 \beta \cdot \alpha \cdot \lambda^{2} \\
P\left(N_{2-5} \longrightarrow N_{1-5}\right)=2 \beta \cdot \alpha \cdot \lambda^{2} \\
P\left(N_{3-5} \longrightarrow N_{1-5}\right)=4 \beta \cdot \alpha^{2} \cdot \lambda^{3}
\end{array}\right.
$$

$N_{1-5}$ corrupted:

$$
\left\{\begin{array}{l}
P\left(N_{3-5} \longrightarrow N_{2-5}\right)=\alpha \cdot \lambda+2 \alpha \cdot \beta \cdot \lambda^{2} \\
P\left(N_{2-5} \longrightarrow N_{1-4} \text { and } N_{1-6}\right)=2 \alpha \cdot \beta \cdot \lambda^{2} \\
P\left(N_{3-5} \longrightarrow N_{1-4} \text { and } N_{1-6}\right)=4 \alpha^{2} \cdot \beta \cdot \lambda^{3}
\end{array}\right.
$$

3.3. Load Capacity Model. The hierarchical clustering of farmland WSNs makes the nodes closer to the sink node undertake more communication tasks, and preventing the premature failures of these nodes is the premise of network invulnerability. Due to the gradient distribution of nodes, the inner layers have fewer nodes than the outer layers, and 
the inner-layer nodes with higher load capacities can offset the negative impact of more extra traffic. Therefore, based on the classical load capacity model and the topological properties of constructed farmland WSNs, we address the load capacity model to optimize the distribution of traffic and promote the network capabilities against cascade failures in this section.

The load capacity of a single node is described as follows:

$$
C_{n}=\left(1+\frac{\varepsilon}{n}\right) L_{n}, \quad n=1,2, \ldots, H,
$$

where $C_{n}$ is the capacity of a single node in the $n$th layer, $L_{n}$ is the initial load of a single node of the $n$th layer, $\varepsilon$ is the adjustment coefficient used to regulate the node capacity value, $n$ represents the layer number, and $H$ denotes the total number of layers in the network.

The initial load $L_{n}$ of a single node in the $n$th layer is computed by

$$
L_{n}=\frac{S_{n+1}}{T_{n}}
$$

where $L_{n}$ is the initial load of a single node at the $n$th layer, $S_{n+1}$ is the total initial load of all nodes at the $n+1$ th layer, and $T_{n}$ signifies the total number of nodes in the $n$th layer.

Hence, the load capacity model can be defined as

$$
C_{n}=\left(1+\frac{\varepsilon}{n}\right) \cdot \frac{S_{n+1}}{T_{n}}, \quad n=1,2, \ldots, H .
$$

\section{Analysis on the Invulnerability of Farmland WSNs}

4.1. Simulation Settings. In the simulations, the number of layers of farmland WSNs is set to 5, the interval distance between layers is $15 \mathrm{~m}$, and the sink node is placed at the center of the simulation area. A total of 200 nodes are distributed in the simulation area. We configure the initial traffic of each node to be 1 unit and the communication reliability $\lambda$ to be 0.8 . The transfer probability of radial link $\alpha$ and the transfer probability of transverse link $\beta$ are initially set to 0.6 and 0.2 , respectively. Each node in the initial network is in a normal state before attacks. In our model, we assume that cascading failures are triggered by the faulty nodes caused by overload of load capacity. All the simulation results are the average of 50 simulations.

To assess the performance of mitigation of cascading failures for the built farmland WSNs, the number of simulation rounds and network efficiency ratio are adopted as evaluation metrics:

(1) A simulation round is defined as the period for all normal nodes to complete data transmissions to the sink node. The number of simulation rounds while the termination condition of node failure rate is satisfied can be expressed as $R$.

(2) Network efficiency ratio is applied to measure the damage degree of cascading failures and can be achieved by

$$
M=\frac{E_{\text {normal }}}{E_{\text {initial }}}=\frac{\left(1 / N_{\text {normal }}\left(N_{\text {normal }}-1\right)\right) \sum_{i \neq j} 1 / d_{i j}^{\prime}}{\left(1 / N_{\text {initial }}\left(N_{\text {initial }}-1\right)\right) \sum_{i \neq j} 1 / d_{i j}},
$$

where $E_{\text {initial }}$ and $E_{\text {normal }}$ represent the network efficiency before and after network damage, respectively; $N_{\text {normal }}$ is the number of normal nodes, and $N_{\text {initial }}$ is the total number of nodes in the initial network; and $d_{i j}$ and $d_{i j}^{\prime}$ denote the number of hops along the shortest path from node $i$ to node $j$ in the initial network and the faulty network, respectively.

4.2. Invulnerability for Random Attacks. Figure 8 depicts the number of simulation rounds $R$ and network efficiency ratio $M$ with varying adjustment coefficient $\varepsilon$ under different levels of node failure rate $\gamma$. From Figure $8(\mathrm{a})$, It can be observed that, with the growth of $\gamma$ from 0.1 to $0.3, R$ raises less with $\varepsilon$ rising from 0 to 40 (average number of nodes per layer) but more with $\gamma$ increasing from 0.4 to 0.9 . In particular, when $\gamma$ increases from 0.7 to $0.9, R$ rises 23.2, 26.8, and 37.5 rounds, respectively. Moreover, when $\varepsilon$ is in the range of 25 to 40 , the trend of $R$ growing with $\varepsilon$ is more obvious. Therefore, to weak the impact of cascading failures, the designer should choose $\varepsilon$ as large as possible. Meanwhile, as $\varepsilon$ stays the same and $\gamma$ grows, the increments in $R$ increase gradually. The performance of inhibiting the spread of cascading failures may benefit from the spiderweb-like topology and communication rules. As is shown in Figure $8(\mathrm{~b})$, when $\gamma$ is within the range $[0.1,0.6]$, with the growth of $\varepsilon$ from 0 to $40, M$ increases by $11.94 \%, 16.35 \%$, $12.31 \%, 23.93 \%, 20.19 \%$, and $27.74 \%$, respectively. $M$ is comparatively small, while $\gamma$ varies from 0.7 to 0.9 , but the rise is up to $53.5 \%$. The results suggest that the farmland WSNs with a higher $\varepsilon$ can lower the damages caused by cascading failures.

Figure 9 demonstrates the relationship between $R$ and $M$, in the case that $\gamma=0.8$. It can be noticed that $M$ continually decreases with the increase of $R$, indicating that $M$ is inversely proportional to $R$. For different $\varepsilon$, when $R$ raises from 0 to $50, M$ rapidly declines from 0.8 to about 0.1 , and the smaller the $\varepsilon$ is, the more notable $M$ reduces. Taking $R=50$ as an example, with the growth of $\varepsilon$ from 0 to $40, M$ decreases by $97.29 \%, 89.48 \%, 88.37 \%, 85.49 \%, 85.54 \%, 85.67 \%$, $85.87 \%, 84.19 \%$, and $84.00 \%$, respectively. Thus, $\varepsilon$ has the function of regulating $M$. However, as $R$ continues to go up from 50, $M$ drops slowly. It can be concluded that, after a certain degree of damage generated by cascading failures, the network scheme for farmland WSNs can improve $R$ with trifling effect on $M$.

4.3. Invulnerability for Deliberate Attacks. Figures $10(\mathrm{a})-10(\mathrm{j})$ show $R$ and $M$ with varying $\varepsilon$ after the failures of the same number of nodes from the 1st layer to the 5 th layer caused by deliberate attacks when $\gamma=0.8$, respectively. As shown in Figures 10(a), 10(c), 10(e), 10(g), and $10(\mathrm{i})$, it can be easily discovered that when $\varepsilon$ is fixed, $R$ declines with the increase in the number of failure nodes for each layer. For instance, when $\varepsilon=10$, the failure nodes in the 3 r $d$ layer grow from 1 to 11 , and $R$ is $69.8,67,61,60.4,57.4$, 


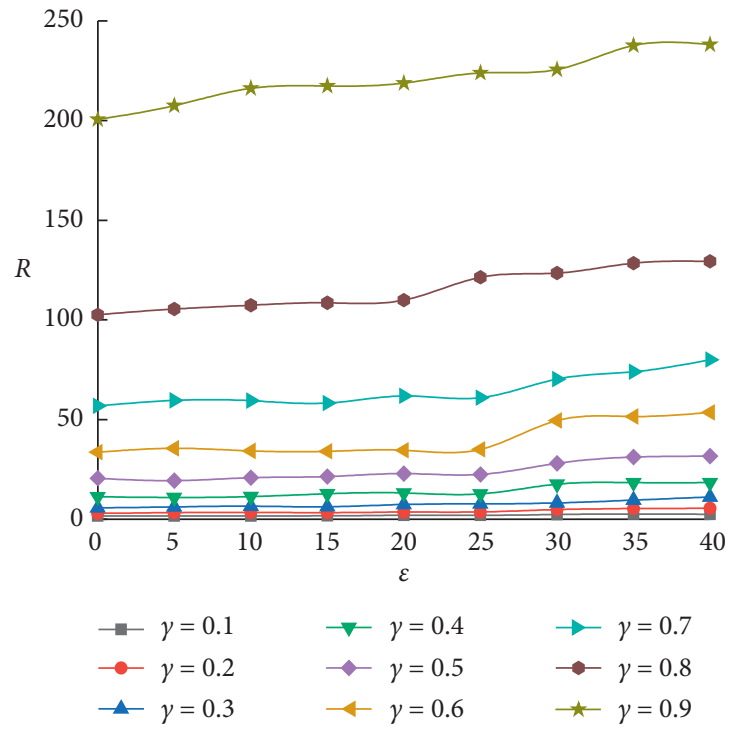

(a)

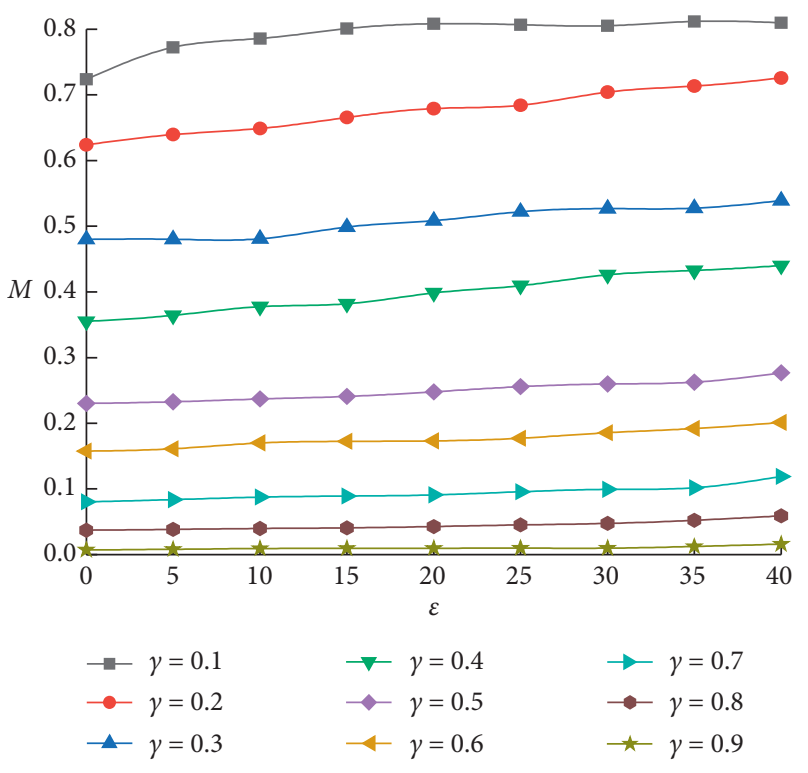

(b)

FIGURE 8: Evaluation metrics with varying $\varepsilon$ for random attacks: (a) number of simulation rounds with varying $\varepsilon$; (b) network efficiency ratio with varying $\varepsilon$.

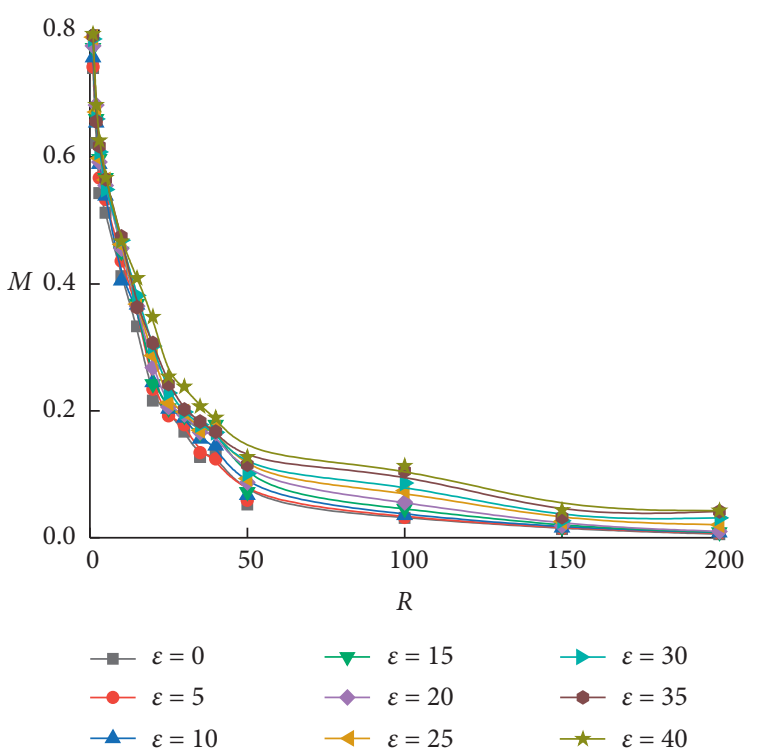

Figure 9: Relevance between $R$ and $M$ for random failure $(\gamma=0.8)$.

and 56.6, respectively. With the growth of $\varepsilon, R$ has an increasing trend under the number of failure nodes is the same. For the 1st layer, in the case that the number of failure nodes is $5, R$ with $\varepsilon$ rising from 0 to 40 is $66.6,66.8,69.4,68.2$, $70,70.2,80.4,86.2$, and 98.8 , respectively. The results infer that the farmland WSNs adopting the network scheme can mitigate the cascading failures in the event of node failures caused by targeted attacks. Furthermore, it can be also observed that when $\varepsilon$ and the number of failure nodes are maintained the same, $R$ reduces more drastically in the inner layers than that in the outer layers. The result shows that $\varepsilon$ has a greater influence on the inner layers by ensuring the load capacity of inner-layer nodes in the spiderweb-like hierarchical topology, and the effect of outer-layer nodes on network invulnerability is less. Besides, $\varepsilon$ is within the range $(30,40)$, and $R$ is improved significantly for the different numbers of failure nodes in each layer. Taking the 2 nd layer as an instance, when $\varepsilon$ is in this range, the average increment of $R$ for each number of failure nodes reaches $16.26 \%$, $24.01 \%$, and $32.04 \%$, respectively.

From Figures 10(b), 10(d), 10(f), 10(h) and 10(j), we can note that, under the identical $\varepsilon$, with the rise of the number of failure nodes, the fluctuation of $M$ is insignificant, which is the result of the link backup mechanism in the farmland WSNs to assure network connectivity at small hop costs. Moreover, it can be seen that $M$ boosts with the increase in $\varepsilon$ for each layer under the circumstance that the number of failure nodes stays the same. For example, when the number of failure nodes in the 5th layer is 7 and $\varepsilon$ raises from 0 to $40, M$ is $0.035,0.037,0.046,0.063,0.072,0.086$, $0.113,0.116$, and 0.135 , respectively. The performance of cascading-failure reduction may originate from the balance of load and traffic of nodes with the continuous growth of $\varepsilon$. Meanwhile, with the same $\varepsilon$ and the same number of failure nodes, $M$ in the inner layers is smaller than that in the outer layers. Due to the more limited influence of edge-layer nodes on the shortest paths between nodes, the results are contrary to the variation characteristics of $R$ under the same condition and consistent with the inversely proportional relationship between $R$ and $M$ in the randomfailure simulations.

To distinguish the difference in cascading invulnerability between different degrees of deliberate attacks with varying $\varepsilon$, when $\gamma=0.8$, we investigate the influence of $\varepsilon$ after the failure of $2,4,6,8$, and 10 nodes and 10, 8, 6, 4, and 2 nodes from the 1st layer to the 5 th layer on the evaluation metrics, 


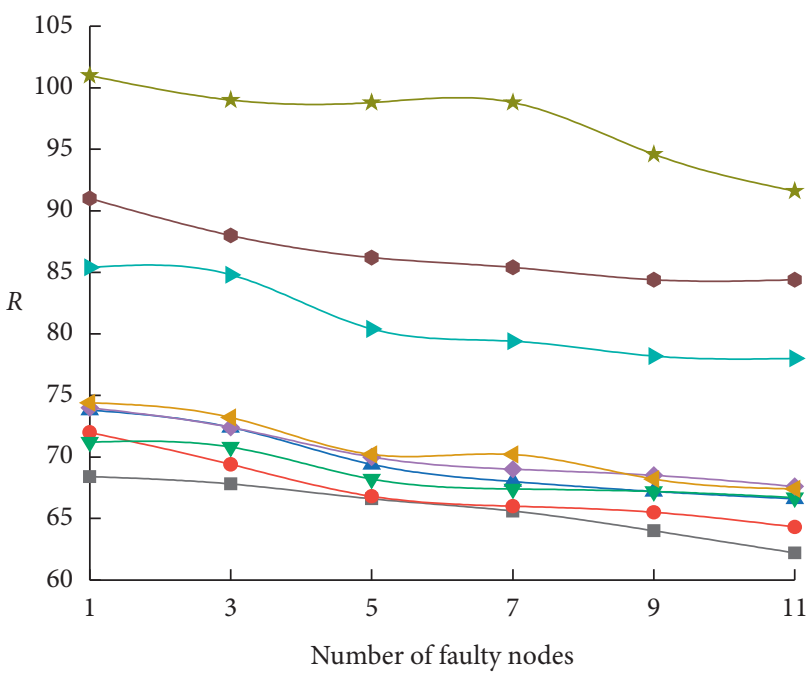

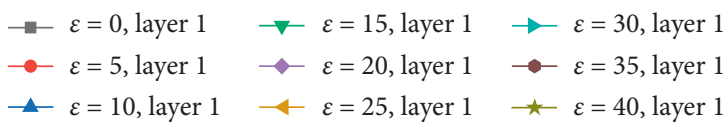

(a)

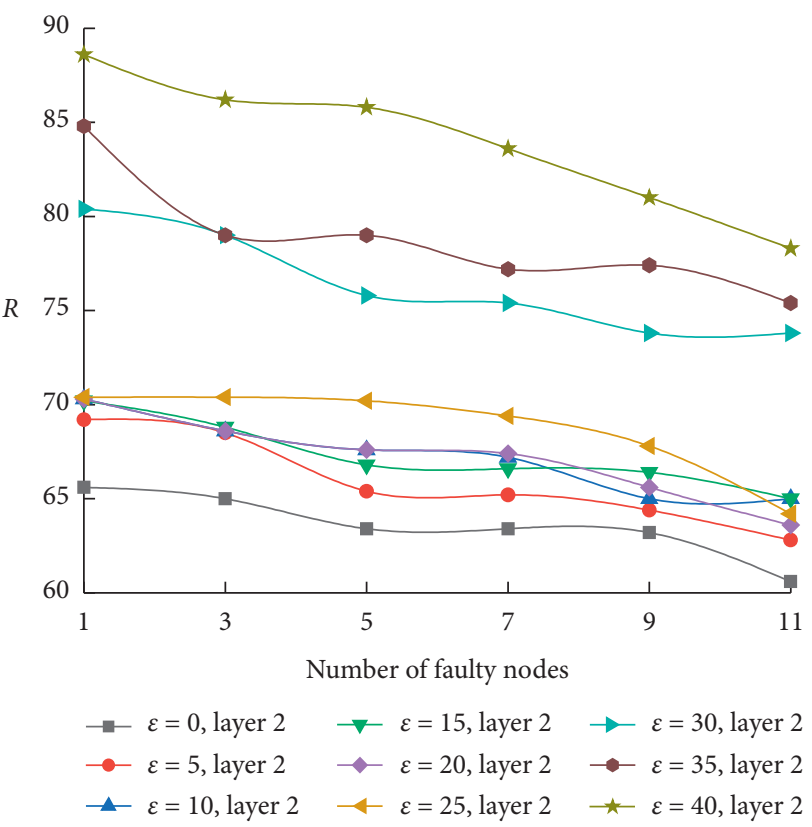

(c)



$\rightarrow \varepsilon=0$, layer $1 \rightarrow \varepsilon=15$, layer $1 \rightarrow \varepsilon=30$, layer 1

$\multimap \varepsilon=5$, layer $1 \multimap \varepsilon=20$, layer $1 \multimap \varepsilon=35$, layer 1

$\multimap \varepsilon=10$, layer $1 \multimap \varepsilon=25$, layer $1 \multimap \varepsilon=40$, layer 1

(b)

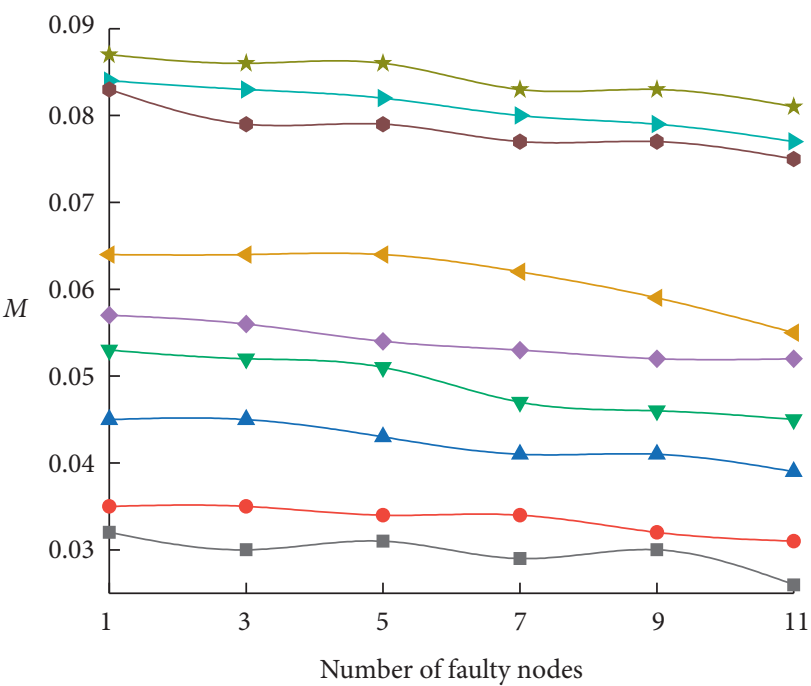

$\neg \varepsilon=0$, layer $2 \rightarrow \varepsilon=15$, layer $2 \rightarrow \varepsilon=30$, layer 2

$\multimap \varepsilon=5$, layer $2 \multimap \varepsilon=20$, layer $2 \multimap \varepsilon=35$, layer 2

$\multimap \varepsilon=10$, layer $2 \multimap \varepsilon=25$, layer $2 \multimap \varepsilon=40$, layer 2

(d)

Figure 10: Continued. 


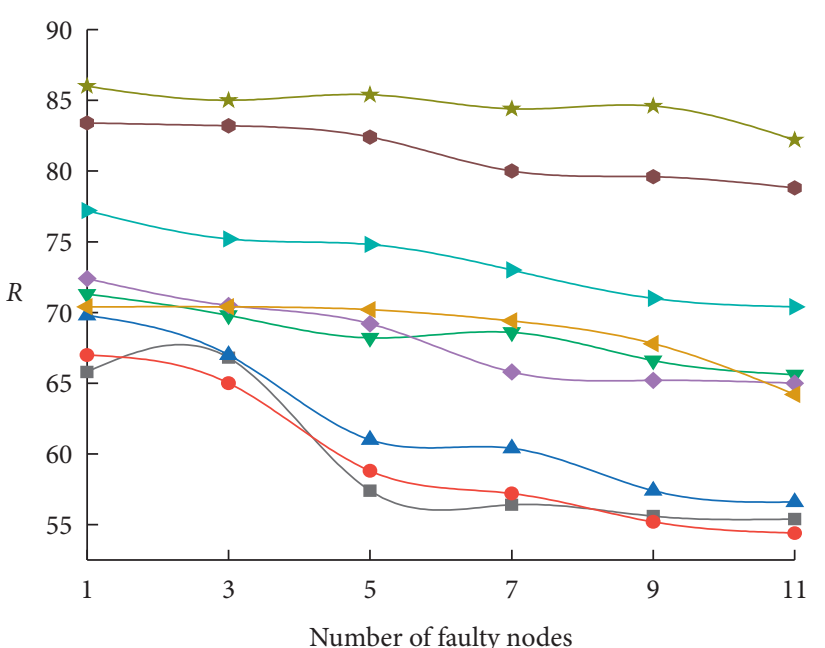

$\multimap \varepsilon=0$, layer $3 \rightarrow \varepsilon=15$, layer $3 \rightarrow \varepsilon=30$, layer 3
$\multimap \varepsilon=5$, layer $3 \multimap \varepsilon$, layer $3 \rightarrow \varepsilon=35$, layer 3
$\multimap \varepsilon=10$, layer $3 \multimap \varepsilon=25$, layer $3 \rightarrow \varepsilon=40$, layer 3

(e)



$\neg \varepsilon=0$, layer $4 \longrightarrow \varepsilon=15$, layer $4 \rightarrow \varepsilon=30$, layer 4

$\multimap \varepsilon=5$, layer $4 \longrightarrow \varepsilon=20$, layer $4 \longrightarrow \varepsilon=35$, layer 4

$\multimap \varepsilon=10$, layer $4 \multimap \varepsilon=25$, layer $4 \quad$ $\leftarrow \varepsilon=40$, layer 4

(g)



$\rightarrow \varepsilon=0$, layer $3 \rightarrow \varepsilon=15$, layer $3 \rightarrow \varepsilon=30$, layer 3

$\multimap \varepsilon=5$, layer $3 \multimap \varepsilon=20$, layer $3 \multimap \varepsilon=35$, layer 3

$\multimap \varepsilon=10$, layer $3 \multimap \varepsilon=25$, layer $3 \multimap \varepsilon=40$, layer 3

(f)



$\rightarrow \varepsilon=0$, layer $4 \rightarrow \varepsilon=15$, layer $4 \rightarrow \varepsilon=30$, layer 4

$\multimap \varepsilon=5$, layer $4 \multimap \varepsilon=20$, layer $4 \multimap \varepsilon=35$, layer 4

$\dashv \varepsilon=10$, layer $4 \multimap \varepsilon=25$, layer $4 \multimap \varepsilon=40$, layer 4

(h)

Figure 10: Continued. 


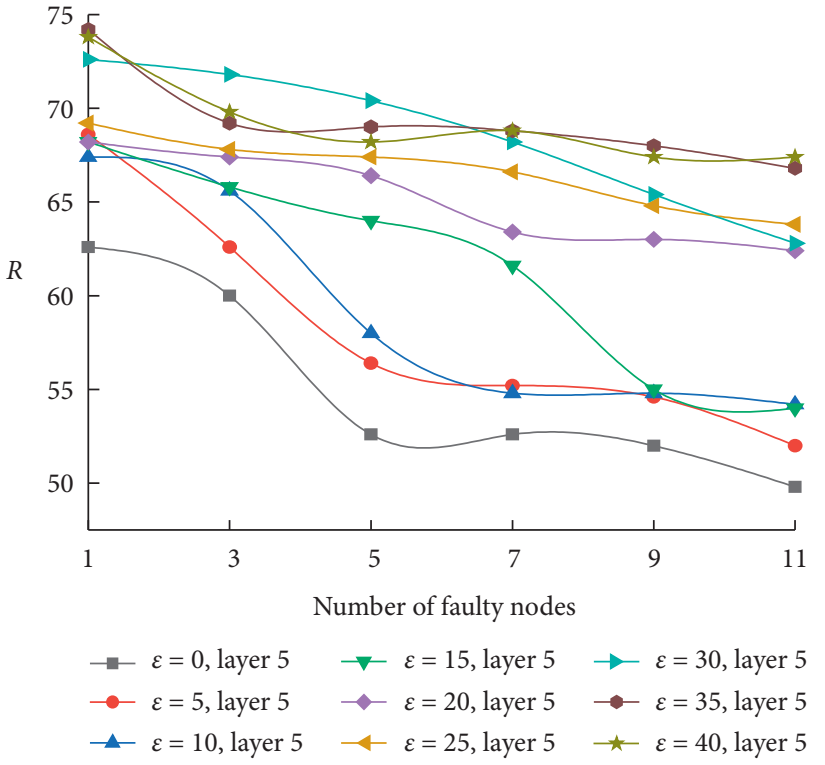

(i)

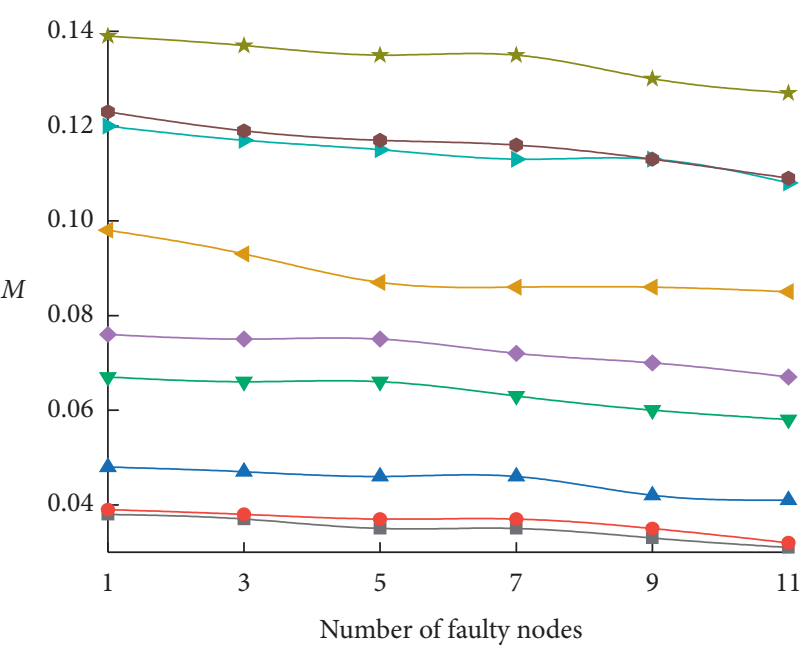

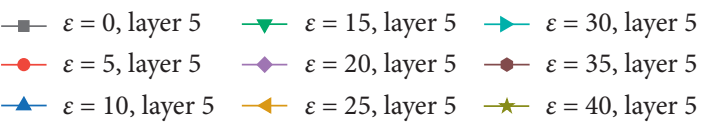

(j)

FiguRE 10: Evaluation metrics with varying $\varepsilon$ for the failures of the same number of nodes in each layer $(\gamma=0.8)$ : (a) $R$ with varying $\varepsilon$ in the 1 st layer; (b) $M$ with varying $\varepsilon$ in the 1 st layer; (c) $R$ with varying $\varepsilon$ in the 2 nd layer; (d) $M$ with varying $\varepsilon$ in the 2 nd layer; (e) $R$ with varying $\varepsilon$ in the 3 rd layer; (f) $M$ with varying $\varepsilon$ in the 3 rd layer; (g) $R$ with varying $\varepsilon$ in the 4 th layer; (h) $M$ with varying $\varepsilon$ in the 4 th layer; (i) $R$ with varying $\varepsilon$ in the 5th layer; (j) $M$ with varying $\varepsilon$ in the 5 th layer.

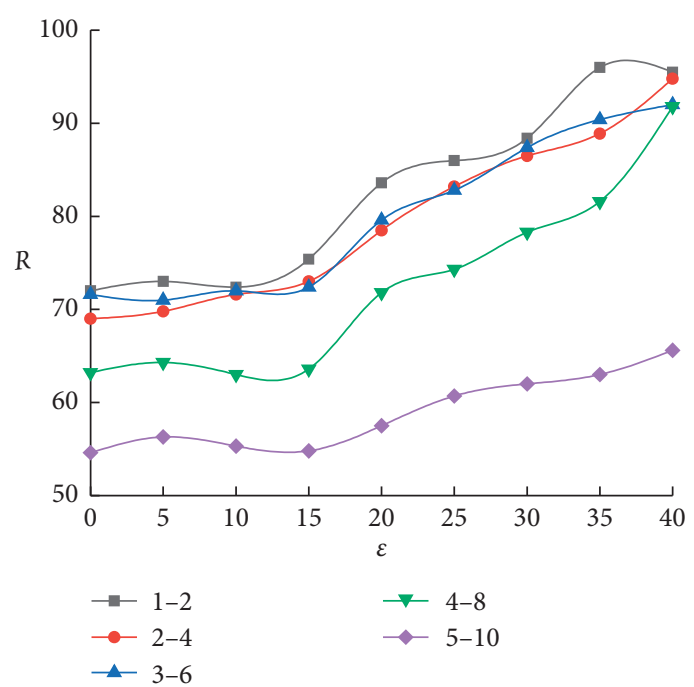

(a)

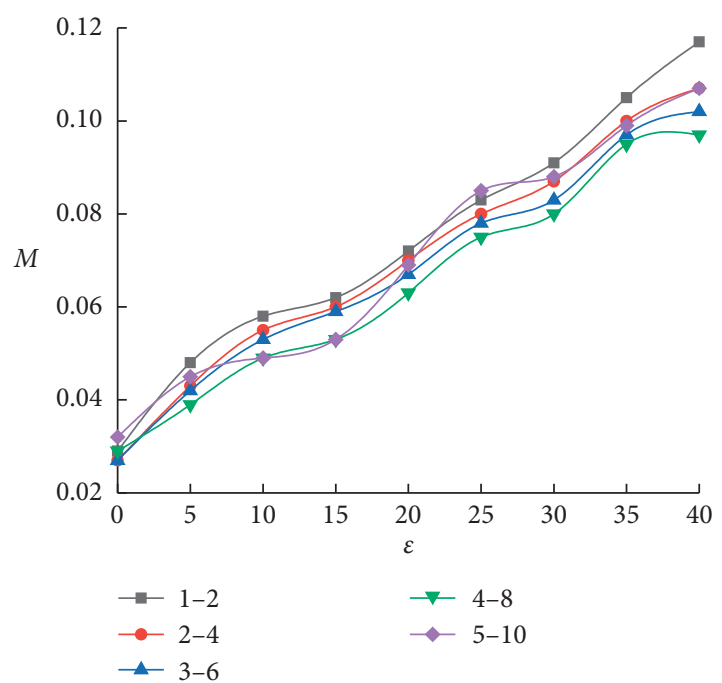

(b)

Figure 11: Continued. 


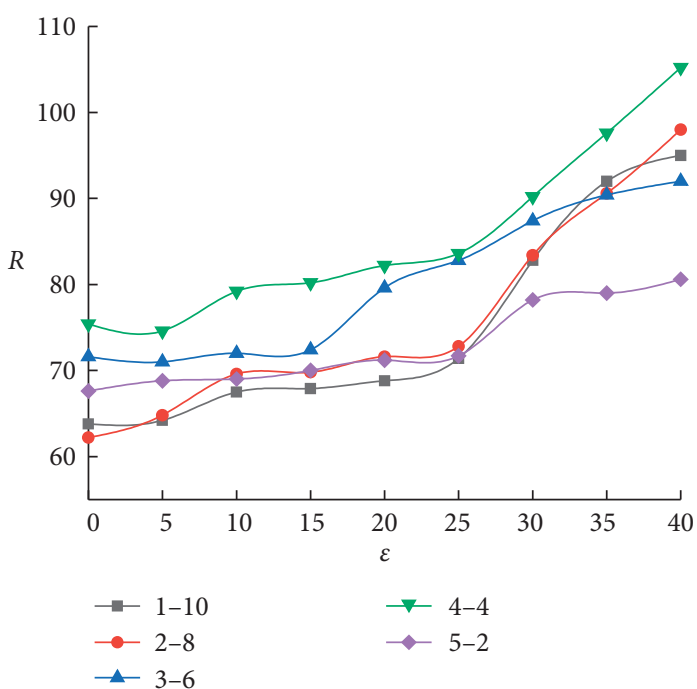

(c)

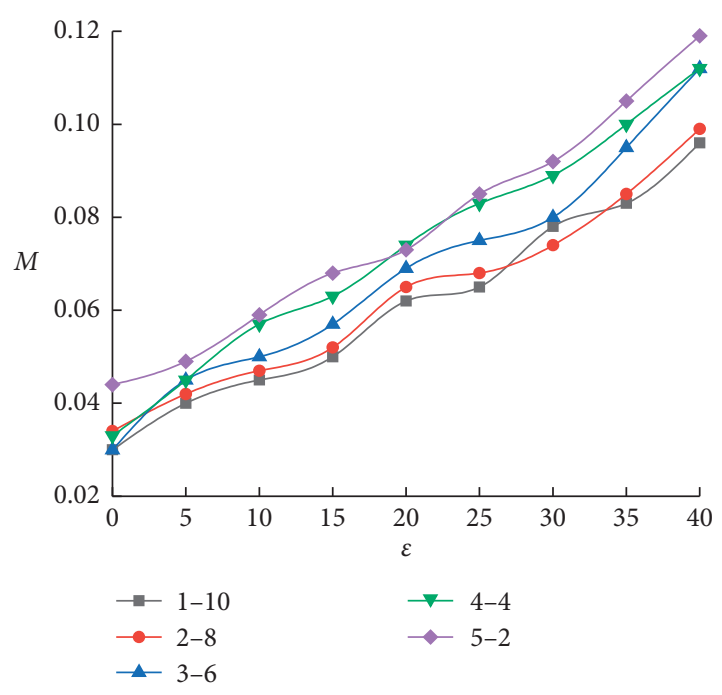

(d)

Figure 11: Comparison of $\varepsilon$ on the evaluation metrics for different degrees of deliberate attacks $(\gamma=0.8)$ : (a) influence of $\varepsilon$ on $R$ after the incremental failures; (b) influence of $\varepsilon$ on $M$ after the incremental failures; (c) influence of $\varepsilon$ on $R$ after the decreasing failures; (d) influence of $\varepsilon$ on $M$ after the decreasing failures.

respectively. As shown in Figures $11(\mathrm{a})$ and $11(\mathrm{c}), R$ demonstrates an upward trend with the increase in $\varepsilon$ for the different number of failure nodes in each layer. When the number of failure nodes rises from the inner layers to the outer layers, compared with that of $\varepsilon=0, R$ under the case of $\varepsilon=40$ increases by $32.63 \%, 37.39 \%, 28.49 \%$, $45.25 \%$, and $20.14 \%$, respectively. While the number of failure nodes reduces from the inner layers to the outer layers, compared with that of $\varepsilon=0, R$ under the circumstance of $\varepsilon=40$ grows by $57.66 \%, 57.55 \%, 28.49 \%, 39.52 \%$, and $19.23 \%$, respectively. It can be discovered that, with the increase in the number of failure nodes in the 1st and 2 nd layers, $R$ raises markedly with the growth of $\varepsilon$. The results manifest that the more the number of failure nodes in the inner layers, the more the $R$ is affected by $\varepsilon$, which is the result of rising the load capacity of the normal nodes in the inner layers. Nevertheless, for the outermost layer, $R$ is lightly affected by $\varepsilon$, and the number of failure nodes slightly impacts the effect of $\varepsilon$ on $R$. Our data imply that the greater $\varepsilon$ in the face of severe deliberate attacks will promote the network invulnerability. From Figures 11(b) and $11(\mathrm{~d})$, when the number of failure nodes increases from the inside out, in the case that $\varepsilon=40, M$ is $3.03,2.96$, $2.77,2.34$, and 2.34 times higher than that of $\varepsilon=0$, respectively. In the condition that the number of failure nodes lessens from inside to outside, compared with the case of $\varepsilon=0, M$ of each layer when $\varepsilon=40$ enhances by 2.20 , $1.91,2.77,2.39$, and 1.70 times, respectively. Therefore, for the two modes of deliberate attacks, $M$ presents a remarkable positive correlation with the increase in $\varepsilon$ and negatively correlates with the number of failure nodes. Moreover, the detection method of deliberate attack should be added in the future to avoid the catastrophic cascading damages induced by a large number of failure nodes.

\section{Conclusions}

Aiming to relieve cascading failures in farmland WSNs, we introduce a bionic research method in this study. On the basis of the destructive vibration testing, the mitigation mechanism of cascade failures of artificial spider web including topological structures, node deployment mode, and link backup is analyzed theoretically. The network scheme consisting of networking methodology, communication rules, and load capacity model is proposed based on the combination of the characteristics of farmland WSNs and the inspirations of artificial spider web. We verified the performance of the developed network scheme by using the number of simulation rounds and network efficiency ratio as evaluation metrics. The results showed that the network scheme achieved the ideal invulnerability for both random and deliberate attacks. The adjustment coefficient in the load capacity model was confirmed for the regulatory effect on network invulnerability. Furthermore, the use of the topology and communication rules inspired by spider web should be advised for lowering the damages of cascading failures. The nodes in the inner layers had a greater impact on the propagation of cascading failures. Our results suggest that the bionic network scheme is an effective approach of cascading-failure mitigation for farmland WSNs.

\section{Data Availability}

The data used to support the findings of this study are available from the corresponding author upon request.

\section{Conflicts of Interest}

The authors declare that there are no conflicts of interest regarding the publication of this article. 


\section{Authors' Contributions}

J.W. conceptualized the study, did formal analysis, gathered resources, performed project administration, and obtained funding acquisition. J.W. and Z.D. formulated methodology and investigated and visualized the study. Z.H. analyzed using software. Z.D. and X.W. validated and supervised the study and reviewed and edited the manuscript. Z.D. and Z.H. curated the data. J.W., Z.D., and X.W. wrote the original draft preparation. All authors have read and agreed to the published version of the manuscript.

\section{Acknowledgments}

This research was funded by the National Natural Science Foundation of China (Grant no. 61771184), Program for Science and Technology Innovation Talents in Universities of Henan Province (Grant no. 20HASTIT029), Program for Innovative Research Team (in Science and Technology) in University of Henan Province (Grant no. 19IRTSTHN021), and Science and Technology Major Project of Henan Province (Grant no. 181100110100).

\section{References}

[1] S. Sharma, R. K. Bansal, and S. Bansal, "Issues and challenges in wireless sensor networks," in Proceedings of the 2013 International Conference on Machine Intelligence and Research Advancement, pp. 58-62, IEEE, Katra, India, December 2013.

[2] T. Ojha, S. Misra, and N. S. Raghuwanshi, "Wireless sensor networks for agriculture: the state-of-the-art in practice and future challenges," Computers and Electronics in Agriculture, vol. 118, pp. 66-84, 2015.

[3] X. Fu, Y. Yang, and O. Postolache, "Invulnerability of clustering wireless sensor networks against cascading failures," IEEE Systems Journal, vol. 13, no. 2, pp. 1431-1442, 2019.

[4] A. More and V. Raisinghani, "A node failure and batteryaware coverage protocol for wireless sensor networks," Computers \& Electrical Engineering, vol. 64, pp. 200-219, 2017.

[5] D. Chen, X. Ren, Q. Zhang, Y. Zhang, and T. Zhou, "Vital nodes identification in complex networks," Physics Reports, vol. 650, pp. 1-63, 2016.

[6] S. Misra, R. Singh, and S. V. R. Mohan, "Information warfareworthy jamming attack detection mechanism for wireless sensor networks using a fuzzy inference system," Sensors, vol. 10, no. 4, pp. 3444-3479, 2010.

[7] R. Ghanbari, M. Jalili, and X. Yu, "Correlation of cascade failures and centrality measures in complex networks," Future Generation Computer Systems, vol. 83, pp. 390-400, 2018.

[8] Z. Zhao, "Research on invulnerability of wireless sensor networks based on complex network topology structure," International Journal of Online Engineering (iJOE), vol. 13, no. 3, pp. 100-112, 2017.

[9] R.-R. Yin, B. Liu, H.-R. Liu, and Y.-Q. Li, "Research on invulnerability of the random scale-free network against cascading failure," Physica A: Statistical Mechanics and Its Applications, vol. 444, pp. 458-465, 2016.

[10] M. Yuvaraja and M. Sabrigiriraj, "Fault detection and recovery scheme for routing and lifetime enhancement in WSN," Wireless Networks, vol. 23, no. 1, pp. 267-277, 2017.
[11] A. Majdandzic, B. Podobnik, S. V. Buldyrev, D. Y. Kenett, S. Havlin, and H. Eugene Stanley, "Spontaneous recovery in dynamical networks," Nature Physics, vol. 10, no. 1, pp. 34-38, 2014.

[12] O. Osanaiye, A. Alfa, and G. Hancke, "A statistical approach to detect jamming attacks in wireless sensor networks," Sensors, vol. 18, no. 6, Article ID 1691, 2018.

[13] A. E. Motter and Y.-C. Lai, "Cascade-based attacks on complex networks," Physical Review E, vol. 66, no. 6, Article ID 065102, 2002.

[14] Y. Wang, B. Chen, X. Chen, and X. Gao, "Cascading failure model for command and control networks based on an m-order adjacency matrix," Mobile Information Systems, vol. 2018, Article ID 6404136, 11 pages, 2018.

[15] H. M. Jawad, R. Nordin, S. K. Gharghan, A. M. Jawad, and M. Ismail, "Energy-efficient wireless sensor networks for precision agriculture: a review," Sensors, vol. 17, Article ID 1781, 2017.

[16] Y. S. Miao, L. Yuan, H. R. Wu, and Q. X. Li, “Optimization of energy heterogeneous cluster-head selection in farmland WSN," Applied Mechanics and Materials, vol. 441, pp. 1010$1015,2013$.

[17] F. Huang, C. Zhao, F. Li, and H. Wu, "An improved farmland WSN topology based on YG and clustering algorithm," Information Technology Journal, vol. 12, no. 21, pp. 6463-6468, 2013.

[18] F. Huang, C. J. Zhao, F. F. Li, and H. R. Wu, “A WSN routing and sleeping algorithm in agriculture intermittent data collection," Applied Mechanics and Materials, vol. 513-517, pp. 606-611, 2014.

[19] H. Wu, H. Zhu, and Y. Miao, "An energy efficient cluster-head rotation and relay node selection scheme for farmland heterogeneous wireless sensor networks," Wireless Personal Communications, vol. 101, no. 3, pp. 1639-1655, 2018.

[20] Z. Erasmus, X. Liu, and S. M van Ruth, "Study on the relations between hyperspectral images of bananas a path planning method for mobile sink in farmland wireless sensor network," in Proceedings of the 2017 IEEE 2nd Information Technology, Networking, Electronic and Automation Control Conference, pp. 1157-1160, IEEE, Chengdu, China, December 2017.

[21] B.-L. Miao, X.-G. Zhao, S.-Y. Wu, and H. Zhu, "Research on power consumption weighted state machine of farmland WSN node," in Proceedings of the 2012 IEEE 2nd International Conference on Cloud Computing and Intelligence Systems, pp. 1141-1144, IEEE, Hangzhou, China, October 2012.

[22] B. Dou, X. Wang, and S. Zhang, "Robustness of networks against cascading failures," Physica A: Statistical Mechanics and Its Applications, vol. 389, no. 11, pp. 2310-2317, 2010.

[23] Z. Qin, B. G. Compton, J. A. Lewis, and M. J. Buehler, "Structural optimization of 3D-printed synthetic spider webs for high strength," Nature Communications, vol. 6, Article ID 7038, 2015.

[24] A. Canovas, J. Lloret, E. Macias, and A. Suarez, "Web spider defense technique in wireless sensor networks," International Journal of Distributed Sensor Networks, vol. 2014, Article ID 348606, 10 pages, 2014.

[25] R. Zaera, A. Soler, and J. Teus, "Uncovering changes in spider orb-web topology owing to aerodynamic effects," Journal of the Royal Society Interface, vol. 11, Article ID 20140484, 2014.

[26] J. Wang, Z. Du, Z. He, and J. Wang, "Exploring vibration transmission rule of an artificial spider web for potential application in invulnerability of wireless sensor network," Applied Bionics and Biomechanics, vol. 2019, Article ID 5125034, 18 pages, 2019. 
[27] J. Wang, Z. Du, and Z. He, "Quantitative invulnerability analysis of artificial spider-web topology model based on endto-end delay," Wireless Communications and Mobile Computing, vol. 2020, Article ID 4617239, 11 pages, 2020.

[28] D. De Guglielmo, B. Al Nahas, S. Duquennoy, T. Voigt, and G. Anastasi, "Analysis and experimental evaluation of IEEE 802.15.4e TSCH CSMA-CA algorithm," IEEE Transactions on Vehicular Technology, vol. 66, no. 2, pp. 1573-1588, 2017.

[29] Y. Zhang and Y. I. Wu, "Multiple sources localization by the WSN using the direction-of-arrivals classified by the genetic algorithm," IEEE Access, vol. 7, pp. 173626-173635, 2019. 